\title{
The Origin and Early Development of Chinese Warp Twisted Fabric Structure
}

\author{
Ziqi Wang ${ }^{1}$, Kehui Deng ${ }^{1}, \&$ Zeyang Peng ${ }^{1}$ \\ ${ }^{1}$ College of Humanities, Donghua University, Shanghai, China \\ Correspondence: Ziqi Wang, College of Humanities, Donghua University, Shanghai, China. E-mail: \\ ziqi_wong@163.com
}

Received: January 4, 2021

Accepted: January 12, $2021 \quad$ Online Published: January 31, 2021

doi:10.5539/ass.v17n2p90

URL: https://doi.org/10.5539/ass.v17n2p90

Fund Project: Doctoral Innovation Fund of Donghua University (Project Number: 17D311001).

\begin{abstract}
The origin of textiles is often associated with braid. Both braids and warp twisted fabrics have twisted structures, but warp twisted fabrics are more advanced and closer to the development direction of textiles.From braids to warp twisted fabrics to various complex fabrics, it is supported by the development of social technology. We sorted and categorized related textiles unearthed in prehistoric times, combined with archaeological records and documentary records that could reflect the production situation at that time to a certain extent, and explored the connection between the origin of warp warp fabrics and the development of social technology through the combination of literature research and physical research.
\end{abstract}

\section{Homologous-Prehistoric Twisted Fabric}

In the Neolithic Age, when the society and technology were very primitive, according to archaeological discoveries, twisted fabrics began to exist in China, and a large number of unearthed remains provide us with proof.

The twisted fabric first appeared at the Caoxieshan site in the Neolithic Age, where three weft twisted fabrics were discovered, woven from wild Kudzu fiber, and were original hand-woven fabric.
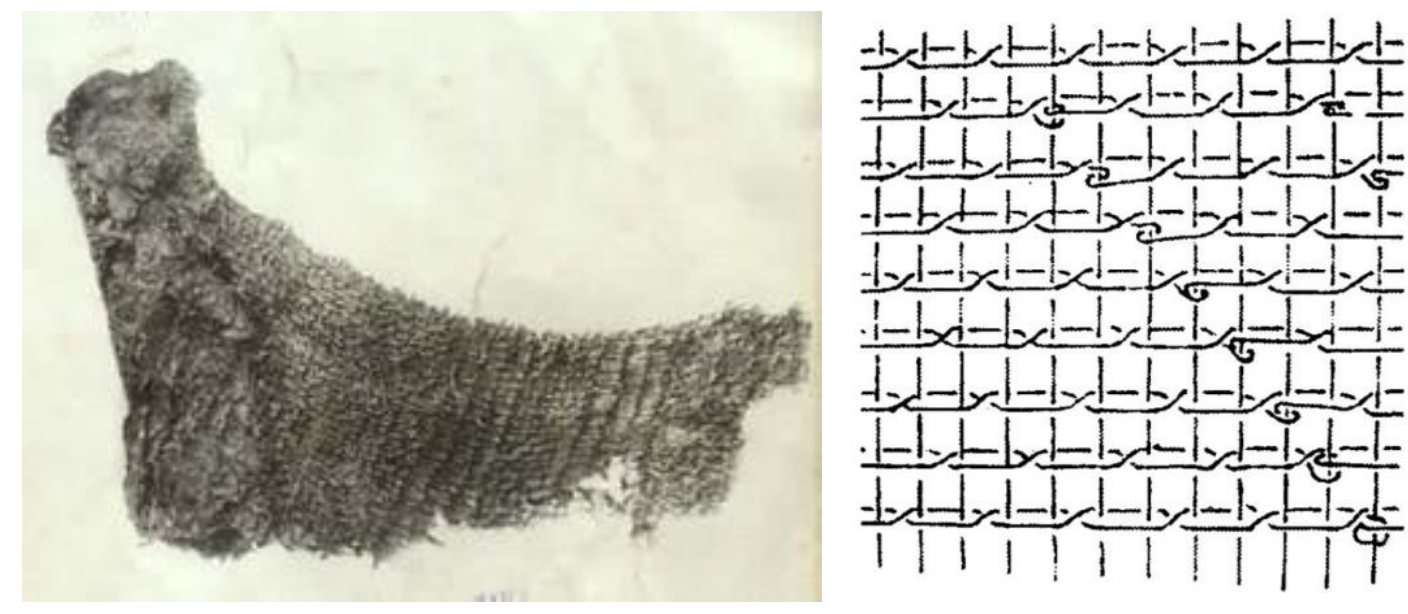

Figure 1. The weft twisted kudzu fabric and its structure unearthed from the Caoxieshan site

A warp twisted fabric belonging to the Yangshao culture period was discovered at the Qingtai site in Xingyang, Henan. It is made of two sets of warp yarns twisted left and right with silk. The remains of spinning wheels, bone needles, and pottery daggers have also been excavated at the site, which may be related to the weaving of twisted fabrics at that time. 


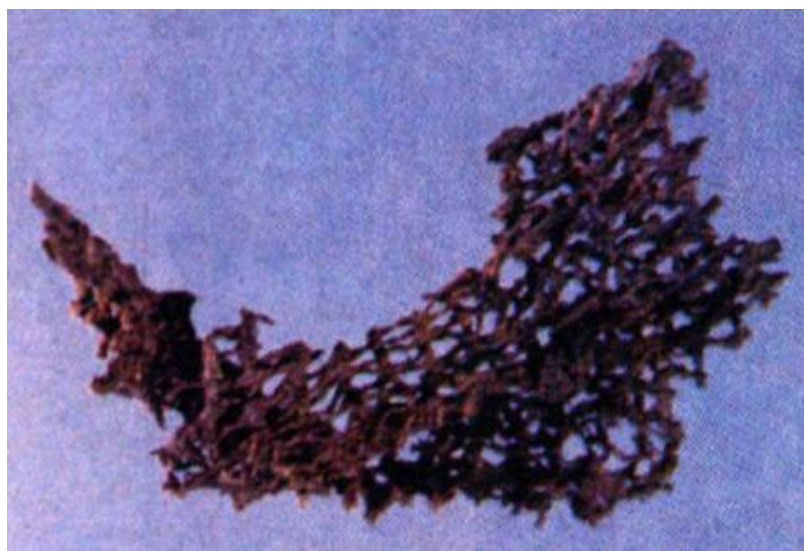

Figure 2. The warp twisted fabric unearthed from the Qingtai site

In the Qianshanyang site in Huzhou, Zhejiang, a four-warp twisted ribbon made of silk was found. Its structure was complex, indicating that the weaving technology at that time had developed to a higher level.

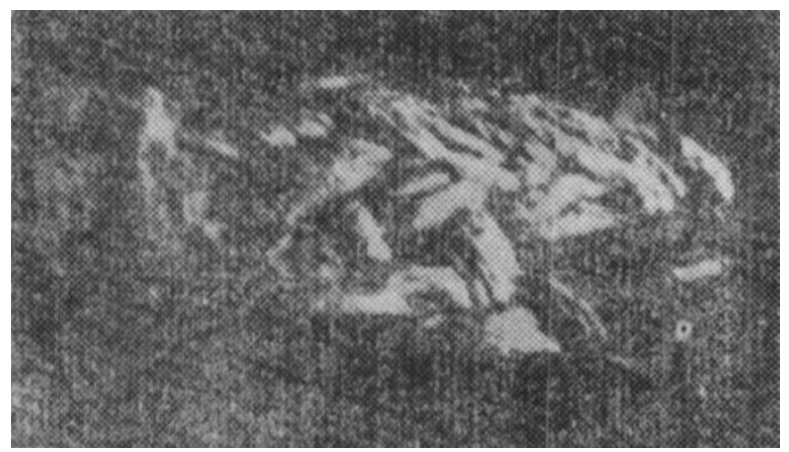

Figure 3. Four-warp twisted ribbon unearthed from Qianshanyang site

From the prehistoric twisted fabrics that have been discovered, it can be found that the weaving process between them is difficult to distinguish. In the manual weaving process, a piece of fabric may contain different processes such as warp twisting and weft twisting.

\section{The Differentiation of Twisted Fabric and the Development of Warp Twisted Fabric}

\subsection{Braid-stronger and More Stable}

Part of the twisted fabric developed in the direction of the braid. Braid fabric is a typical handmade fabric, usually twisted with weft yarns. This type of fabric is developing towards a strong and stable practicality, and is mostly used in webbing and shoes.
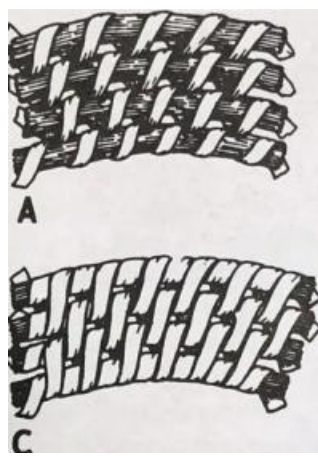
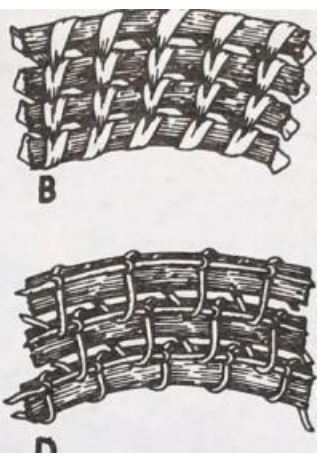

Figure 4. Common braid structure

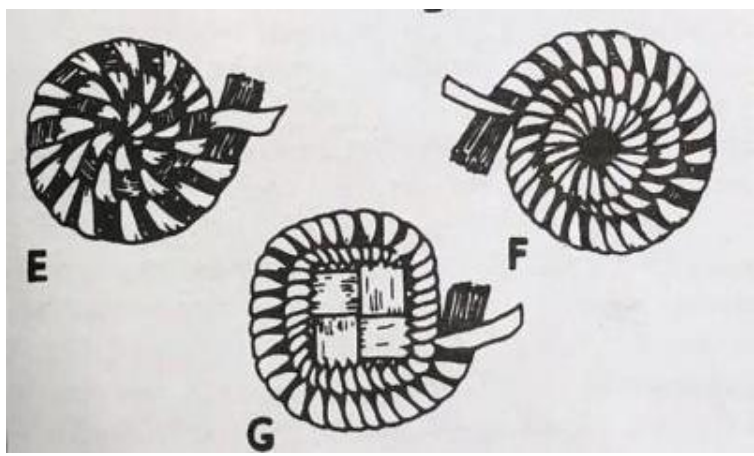

A small piece of braid was found in the early Shang Dynasty site at Fengxia, Beipiao, Liaoning Province. The structure of the weft yarn is straight, and the warp yarn is twisted up and down in a group of two.

The Han Dynasty braided belt was unearthed at the Xuanquanzhi site in Dunhuang, Gansu. The braided belt yarns are all diagonally $45^{\circ}$, and the two directions are twisted and the structure is clear. 


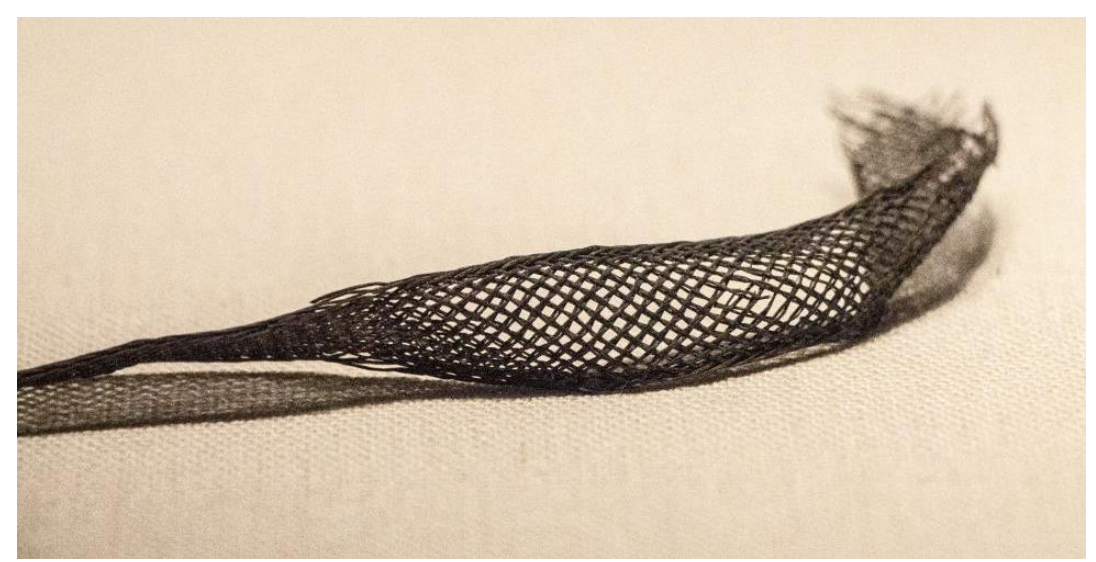

Figure 5. Han Dynasty braided belt unearthed in Xuanquanzhi site

Braided shoes mainly appear in Xinjiang. For example, the "Yisunyi" twisted shoes unearthed in Yingpan during the Wei and Jin Dynasties were made by twisting hemp as the raw material.

\subsection{The Warp Twisted Fabric Officially Appeared - from the Yin Dynasty}

From the Yin Dynasty, typical simple two-warp twisted fabric began to appear, which was a milestone in the development of warp twisted fabrics.

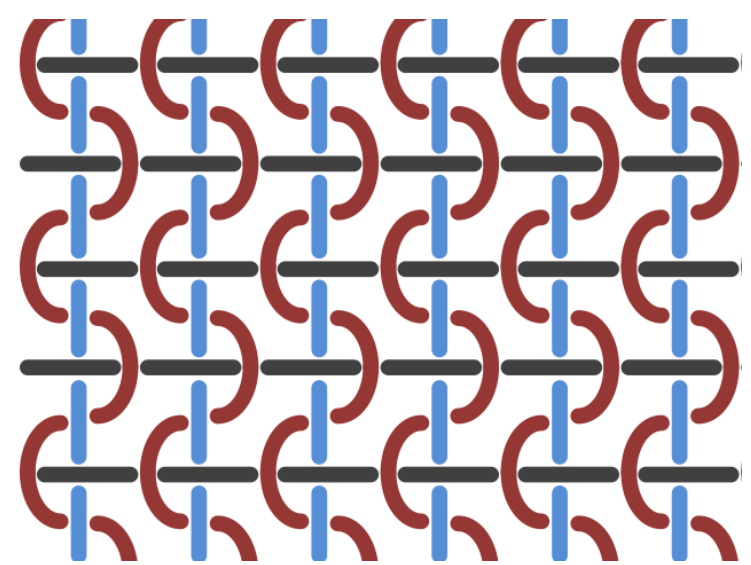

Figure 6. Structure of typical simple two-warp twisted fabric

Two pieces of warp twisted fabric were unearthed from the tomb of Fuhao in Yinxu, with larger holes. As far as we know, this is the earliest warp twisted fabric specimen produced on a loom in China.
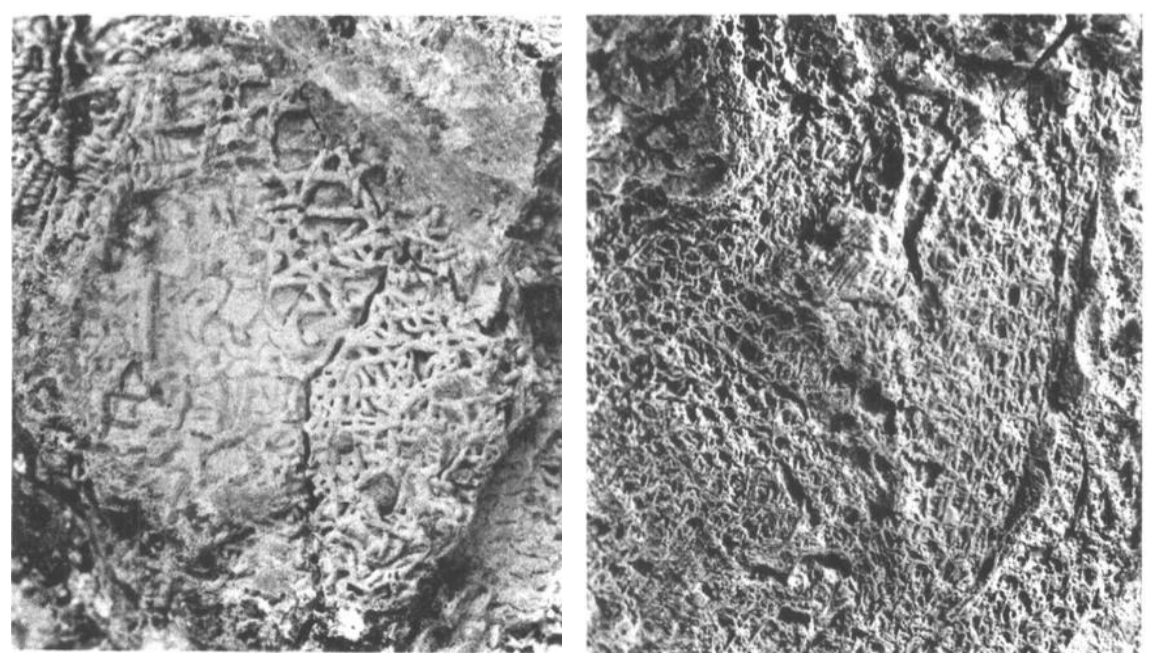

Figure 7. Warp twisted fabric remains in the tomb of Fuhao

Remains of warp twisted silk fabrics were also found in the Shang Dynasty ruins in Taixi Village, Gaocheng, Hebei. The fabric is attached to a copper goblet, which is a simple two-warp twisted fabric. 
There are many discoveries about this type of simple two-warp twisted fabric. For example, in the Guozhong Tomb in Shangcunling, Henan, the Wulipai Chu Tomb in Changsha, the Zuojiatang Chu Tomb in Changsha, and the Langjiazhuang Tomb of Eastern Zhou Dynasty in Linzi, Shandong, similar fabrics appeared. This series of discoveries shows that the structure of the twisted warp fabric of the Zhou Dynasty gradually stabilized.

\subsection{Warp Twisted Fabric with Complex Structure-from the Middle of Zhou Dynasty}

In the middle of Zhou Dynasty, warp twisted fabrics developed by leaps and bounds.

Simple twisted warp fabrics and patterned twisted warp fabrics from the Zhou Dynasty were found in the Palace of Leifeng Pagoda in Hangzhou. The jacquard twisted warp fabric has officially appeared.

In the 3rd century BC, a large number of silk fabrics were unearthed from the Jiangling Chu Tomb in Mashan, Hubei, among which four warp twisted fabrics appeared. The structure of this fabric is much more complicated than the previous simple fabric with two warps twisted together.

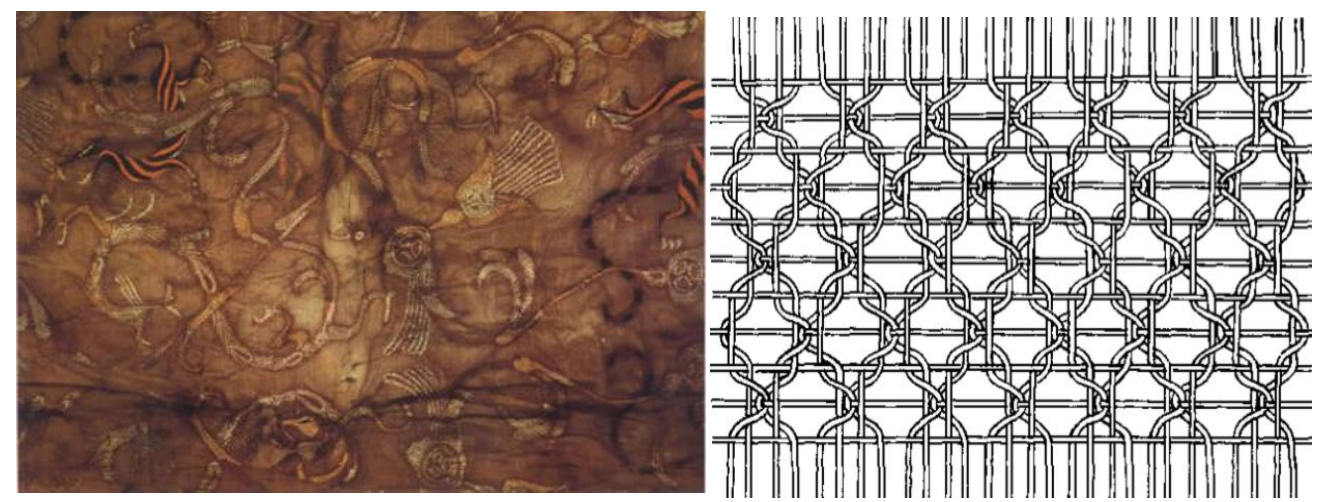

Figure 8. The four-warp twisted fabric and its structure unearthed from the Jiangling Chu Tomb

The warp twisted fabric of the early Western Han Dynasty was found in the Anji Chu Tomb in Zhejiang. It is a jacquard four-warp twisted fabric, and its delicate diamond pattern is clearly visible.
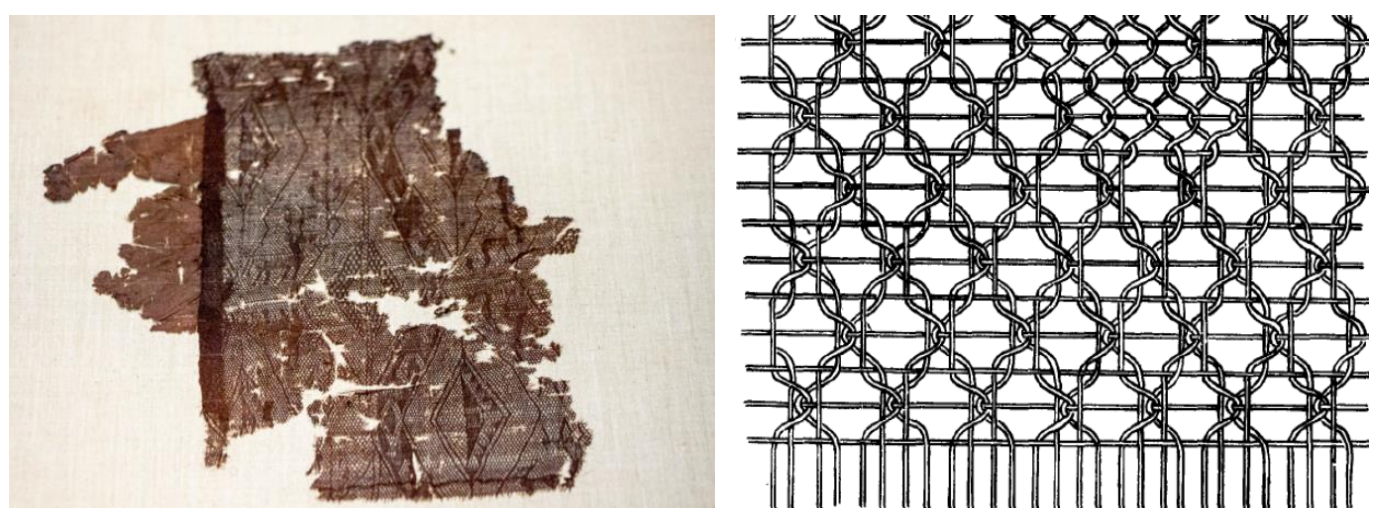

Figure 9. The four-warp twisted jacquard fabric and its structure unearthed from Anji Chu Tomb
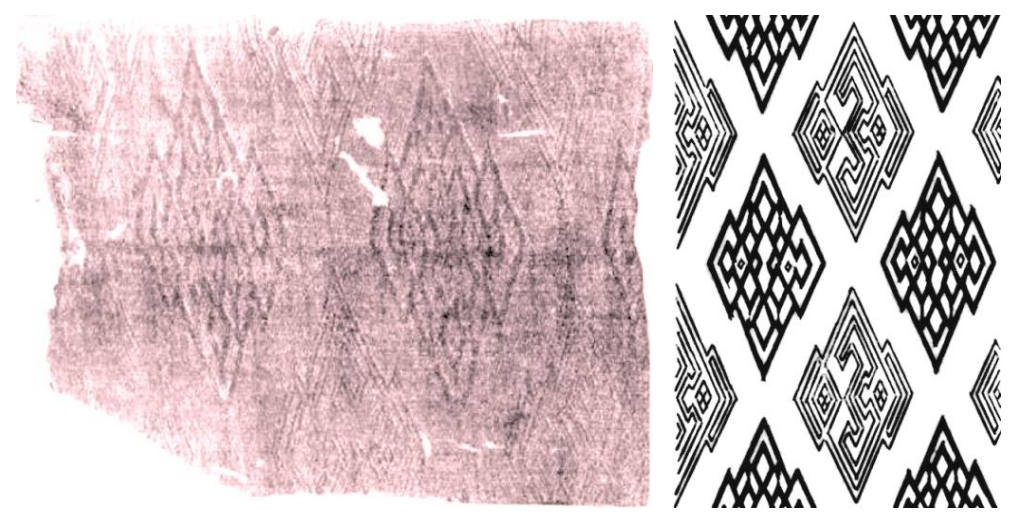

Figure 10. N18 diamond twisted warp fabric and its pattern unearthed from the Mawangdui Han tomb 
A large number of warp twisted fabrics were unearthed from the Mawangdui Han Tomb in Changsha in 166 BC. Among them, there are several diamond-patterned jacquard four-warp twisted fabrics. The red fabric numbered N18 is the most famous. The pattern uses thick and thin lines to form a diamond pattern, one row of thick patterns and one row of fine patterns alternate, and the fabric is dense and delicate.

Similar diamond jacquard warp twisted fabrics were found in other tombs in the Han Dynasty. For example, the Fenghuang Mountain Han Tomb of in Jiangling, Hubei, the Wangxu Tomb of the Eastern Han Dynasty found in North Korea, Mozuizi Han Tomb in Wuwei, Gansu, Mancheng Tomb in Hebei, and Noinwula Tomb in Mongolia.

A piece of four-warp twisted horizontal fabric from the Han Dynasty was unearthed from the Fengsui site in Matuwan, Dunhuang, Gansu. This fabric is composed of three wefts added to the four-warp twisted fabric to give the fabric a horizontal stripe effect.
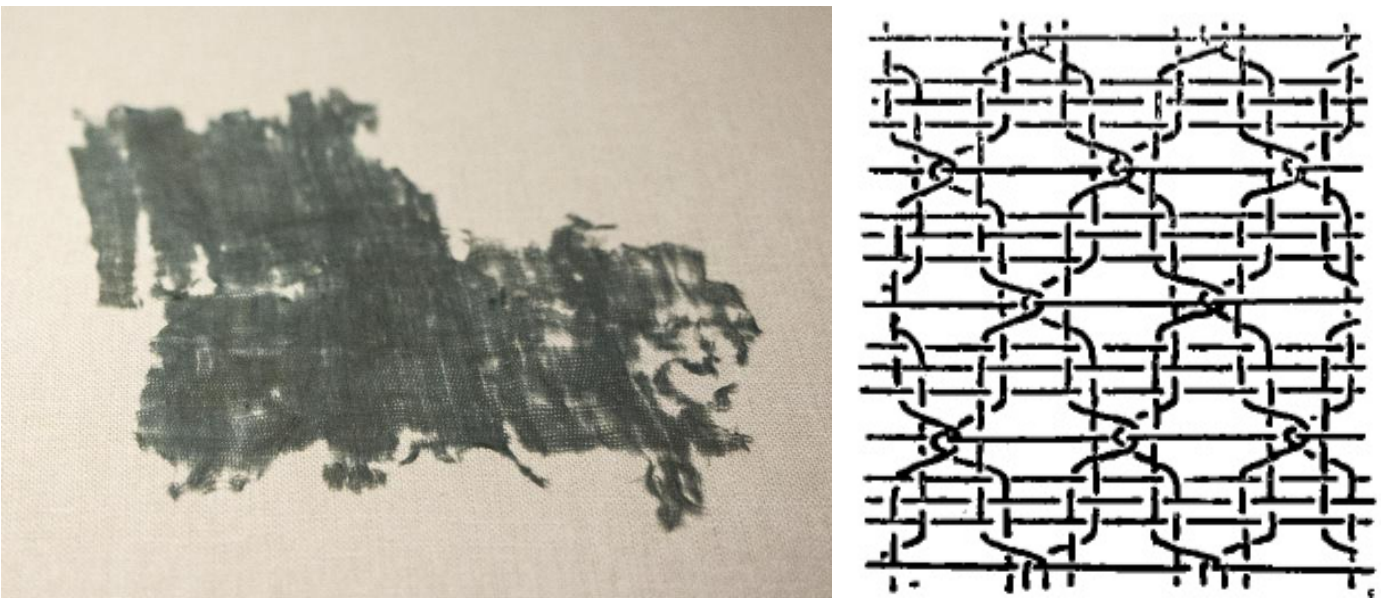

Figure 11. The four-warp twisted horizontal fabric and its structure unearthed from the Fengsui site

The two-warp twisted fabric of the Han Dynasty has also been continued. A simple two-warp twisted fabric was unearthed from the Niya site in Minfeng, Xinjiang, and a jacquard two-warp twisted wool fabric was unearthed from the Fengsui site in Matuwan, Dunhuang, Gansu.

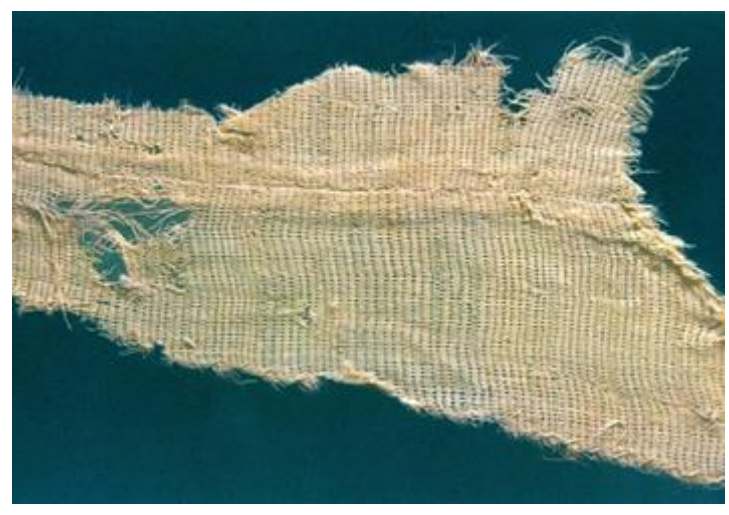

Figure 12. Simple two-warp twisted fabric unearthed form the Niya site

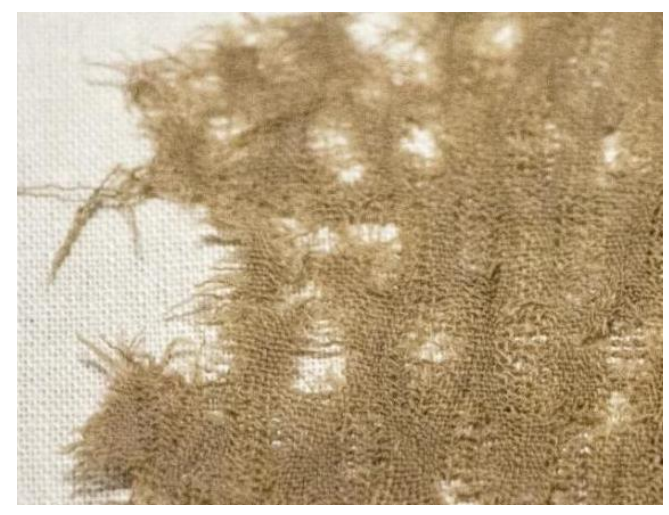

Figure 13. Jacquard two-warp fabric unearthed from the Fengsui site

\subsection{Variety of Patterns-Sui and Tang Dynasty}

A large number of four-warp twisted fabrics from the Sui and Tang dynasties were unearthed in Turpan, Xinjiang. The patterns are different, such as overlapping diamond patterns, diagonal grids, dotted flowers, stripes, etc.

A piece of jacquard two-warp twisted fabric from the late Tang Dynasty was found in Dunhuang Mogao Grottoes. The pattern is composed of continuous squares and swastika patterns. 


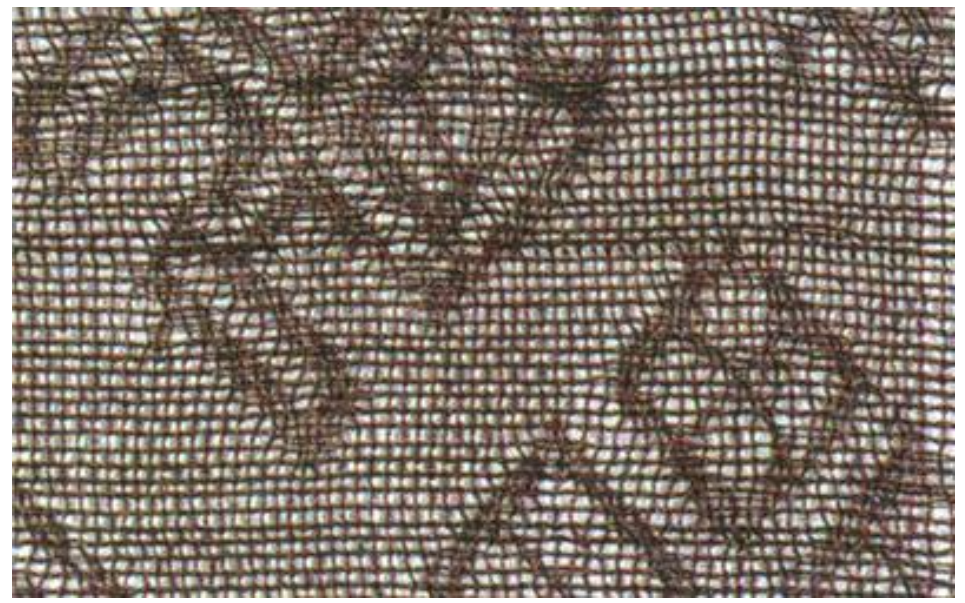

Figure 14. Jacquard two-warp twisted fabric found in Dunhuang Mogao Grottoes

A series of exquisite warp twisted fabrics were unearthed in Astana, Xinjiang. Including a set of colorful jacquard four-warp twisted fabric with various geometric patterns and rich colors. There are also several printed twisted warp fabrics. One has a grape pattern printed on the white warp twisted fabric, and the other has a white pattern printed on the dark green warp twisted fabric.

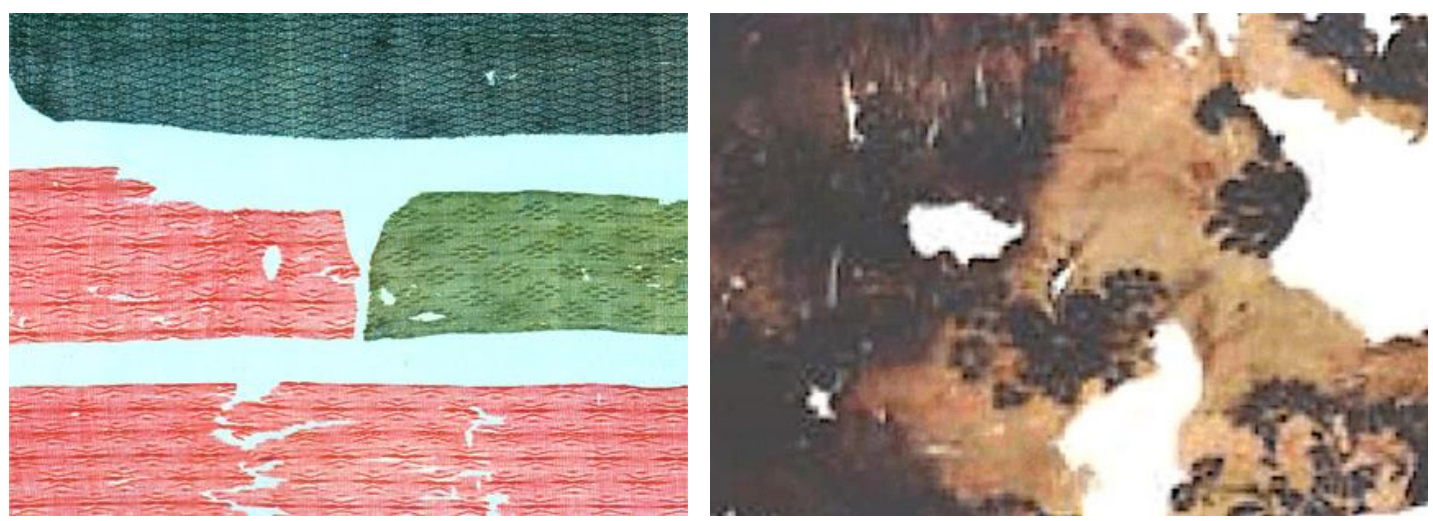

Figure 15. Colored jacquard and white grape pattern warp twisted fabric in Astana

\section{Analysis of the Reasons for the Development of Warp Fabrics}

From the above information, we can summarize the origin and early development of Chinese warp twisted fabrics. The warp twisted fabric has sprouted in prehistoric times, but it is mixed with the braid and cannot be clearly distinguished. The typical two-warp twisted fabric woven by a loom began to appear in the Yin Dynasty. Since the middle of the Zhou Dynasty, the structure of warp twisted fabrics has become diversified, with diamond-patterned jacquard four-warp twisted fabric as a typical representative. During the Sui and Tang Dynasties, the patterns of warp twisted fabrics began to diversify, with various geometric patterns and realistic patterns appearing.

The development of warp twisted fabrics in the early history was driven by a variety of factors, including internal factors for the development of the fabric itself, and external factors such as weaving raw materials, government management, and loom manufacturing.

\subsection{Perfection of the Fabric's Own Structure}

The internal factor of the development of twisted warp fabrics is to form a more stable and beautiful fabric through continuous improvement of the structure, and the development path is well-documented.

In prehistoric twisted fabrics, the warp threads may be connected to the left and right with fingers, without the participation of weft threads. Only the warp yarns are twisted to form a simple net-like fabric. This structure similar to a fishing net makes the fabric very easy to shrink in the horizontal direction.

In order to stabilize the fabric structure, weft threads are added to the net-like fabric to become the original two-warp twisted fabric, which can strengthen the transverse connection of the fabric. With the support of the weft yarns, the twisted warp fabric has a relatively stable planar structure, making it difficult to deform when subjected to force. 
In order to facilitate the use of looms, the warp yarns are grouped into groups to become a typical two-warp twisted fabric in the Yin Dynasty. Continue to weave multiple weft yarns, the fabric can show horizontal stripes.
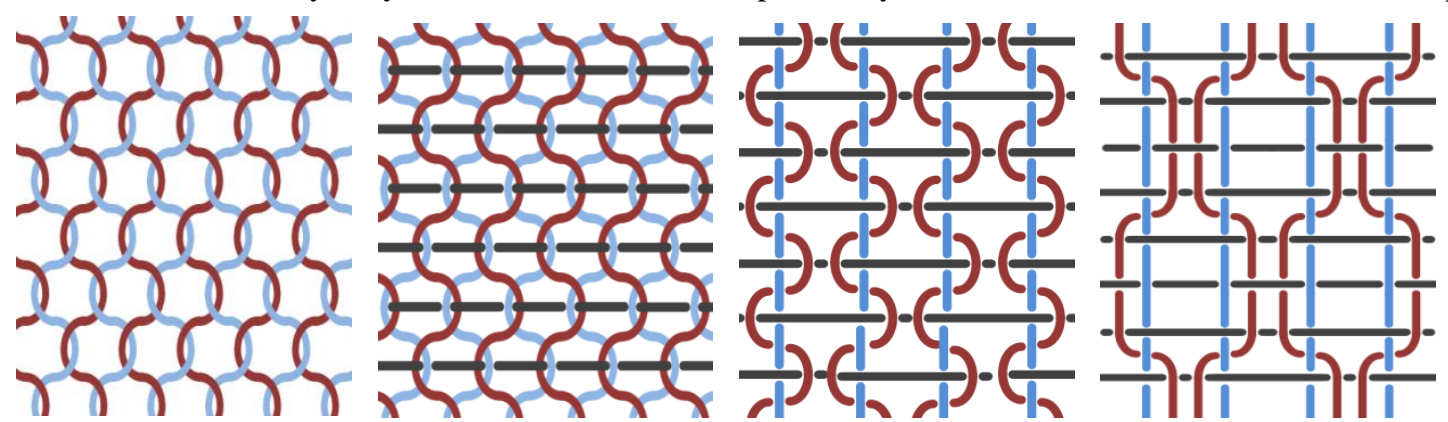

Figure 16. The structure evolution of two-warp twisted fabric

The other direction is the development of complex network structures. Through the compound between the warp yarns, a four-warp twisted fabric was developed. Each group of four adjacent warp threads forms a hexagonal hole.

The four-warp twisted fabric can also be added with wefts to make it show horizontal visual effects. It can also form complex patterns by changing the warp twist rules.
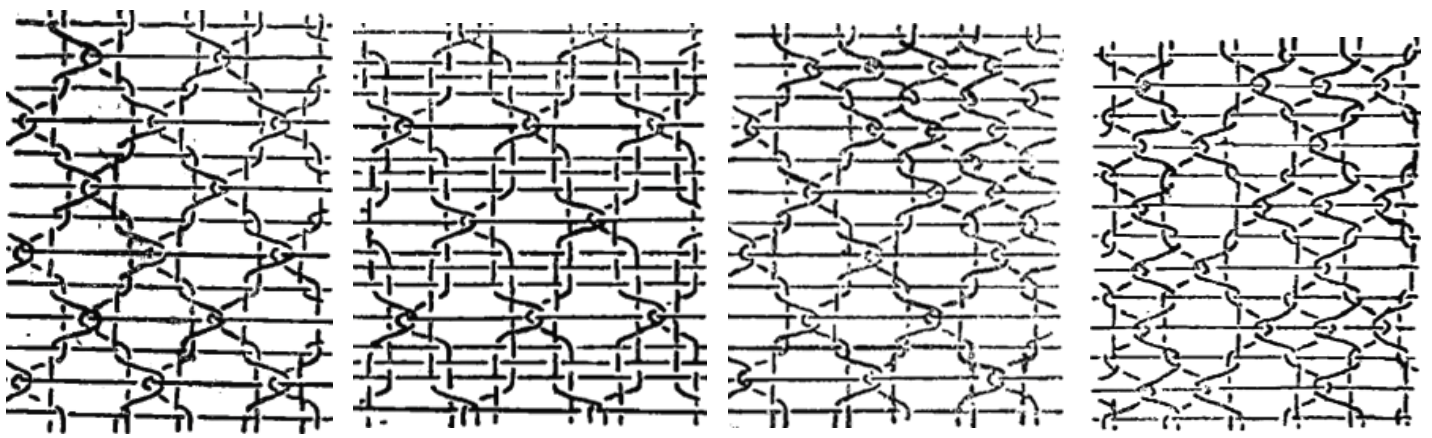

Figure 16. The structure evolution of four-warp twisted fabric

Through the changes of warp and weft in the warp twisted fabric structure, the organization of twisted warp fabrics has continuously developed new varieties, which has achieved the diversity of twisted warp fabrics in the early history of history.

\subsection{Changes in Mainstream Textile Raw Materials}

In prehistoric times, the fabric was mainly woven by hand, and the performance requirements of the yarn were low. Pueraria, hemp, and untwisted silk could all be woven. Among them, pueraria and hemp fibers were easier to obtain and became common textile raw materials at that time.

China is the first country in the world to invent sericulture, silk reeling and silk weaving. The half a silkworm cocoon found at the Neolithic site in Xiyin Village, Shanxi is the earliest relic related to mulberry silk weaving.

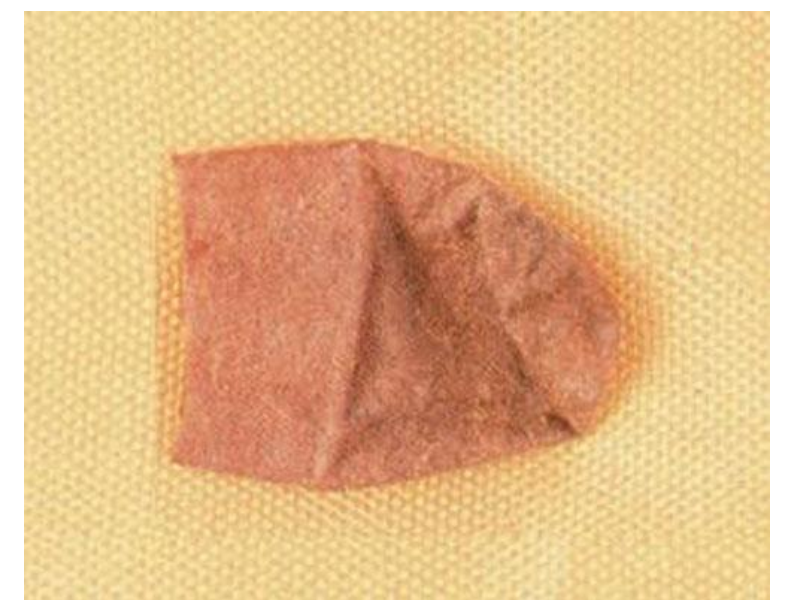

Figure 18. Half a silkworm cocoon unearthed in Xiyin Village 
Since the Yin Dynasty, silk has become a common weaving material. The geographical and climatic conditions for mulberry and sericulture in the Yangtze River Basin are relatively superior, where the silk industry has risen early, developed rapidly and has a high level

The widespread use of silk makes it possible to produce warp twisted fabrics on looms. To produce warp twisted fabrics, stronger filaments are needed, and the strength of Pueraria and hemp yarn is far less than that of silk of the same specification. With the popularity of silk, the production of warp twisted fabrics has also been developed.

\subsection{Standardized Government Management}

On the one hand, the government attaches great importance to the production of silk, which promotes the production of warp twisted fabrics.

During the Eastern Zhou Dynasty, various places paid more attention to sericulture. The government ordered mulberry trees to be planted around each courtyard, and even large areas of mulberry trees were planted in the wild to raise silkworms.

The Yin Dynasty had full-time officials to supervise the production of silk thread and inspect the quality of silk thread. In the Yinxu inscriptions, there are several records of interrupted silk weaving, which shows the importance of silk weaving at that time.

The prosperity of the sericulture industry has greatly improved the quality of silk used in weaving.

On the other hand, the government has set up textile institutions to directly produce twisted warp fabrics.

According to "The Ten Kingdoms of the Spring and Autumn Period", the silk industry was very prosperous at that time. Wuyue Kingdom once presented a large amount of silk fabrics to the Central Plains government, including warp twisted fabrics.

An official seal was unearthed from the Chu tomb in Changsha. Its content shows that in the Warring States period, there was an organization specializing in the management of the textile industry and a government-led weaving system was formed.

In the Tang Dynasty, under the supervision of the government, the textile industry was larger with a finer division of labor, and a weaving workshop for twisted warp fabrics was set up in the capital, which is recorded in the "Tang Liudian".

\subsection{Development of Loom Manufacturing Technology}

The weaving tools unearthed in prehistoric times mainly include spinning wheels and bone needles, which can be used for simple twisted weaving by hand.

In the middle of the Neolithic Age, the original waist loom was unearthed at the Hemudu site in Zhejiang. With the development of weaving technology, the waist loom may have been used to weave two-warp twisted fabric before the Yin Dynasty.

In the Shang Dynasty, the emergence of multi-heald and multi-tangle jacquard looms provided weaving conditions for jacquard warp twisted fabrics.Four warp twisted fabrics appeared from the middle of Zhou Dynasty, and at least two twisted heddles were required to complete the twisting during weaving. It shows that at least it had been woven on a simple loom with twisted healds. It is also possible to weave simple jacquard warp twisted fabrics by installing twisted heddles on the Shang Dynasty jacquard loom.

In the Han Dynasty, a loom with a heddle jacquard mechanism began to weave various silk fabrics. By installing twisted heddles on the jacquard loom, we can weave warp twisted fabrics with variable patterns, which is consistent with the diversified pattern of twisted warp fabrics in the Sui and Tang Dynasties.

\section{Conclusion}

This paper sorts out the origin and development of warp twisted fabrics from prehistoric times to the Tang Dynasty. We clarify the development of warp twisted fabrics in the early history and discussed the reasons for fabric changes.

For the division of development periods, prehistoric is the budding period of twisted warp fabrics, in which the twisted warp fabrics have not yet formed a fixed fabric structure, and the twisted warp structure and the twisted weft structure are mixed together in the process. Simple two-warp twisted fabric woven by a loom officially appeared in the Yin Dynasty, and then there were two obvious developments. From the middle of Zhou Dynasty is the development period of fabric structure, a large number of diamond-patterned four warp twisted fabric 
appeared. The Sui and Tang Dynasties were the period of development of fabric patterns, and various innovative geometric patterns and realistic patterns appeared.

Analyzing the reasons behind the development of warp twisted fabrics, we have summarized four aspects: First, the innovation of twisted weaving methods is the internal cause of the development of warp twisted fabrics and has achieved the diversity of fabric structures. External influences include: Second, the rise of silk enables warp twisted fabrics to be woven on looms. Third, the standardized management of the government directly promoted the production of silk and warp twisted fabrics. Fourth, the development of textile tools promotes the development of fabric structures.

\section{References}

Hubei Province. (1985). Mashan No. 1 Tomb of Chu, Jiangling. Beijing: Cultural Relics Press.

Hui, X., Qiuming, Q., Maosong, L. (1981). Verification of Silk Fabrics Unearthed in Qian Shanyang. Silk, (2), 45-47.

Institute of Archaeology, Chinese Academy of Sciences. (1957). Couch excavation report. Beijing: Science Press.

Institute of Archaeology, Chinese Academy of Social Sciences. (1980). Yinxu Fuhao Tomb. Beijing: Cultural Relics Press.

Jiuling, Z. (1997). Full translation of Tang Liudian. Gansu: Gansu People's Publishing House.

Ke, R. (1995). Looking at several problems in the mulberry, silkworm, silk, and silk industries in the Shang Dynasty from the oracle bone inscriptions. Journal of Soochow University: Engineering Edition, (S2), $47-52$.

Songlin, Z., \& Hanyu, G. (1999). Observation and Research on Silk and Hemp Fabrics Unearthed from Qingtai Site in Xingyang. Original Chinese, (3), 10-16.

Tongying, Z. (2017). Research on the Han and Tang Dynasty dyeing patterns and craftsmanship unearthed in Xinjiang. Journal of Zhejiang Textile and Clothing Vocational and Technical College, 16(62), 82-87. https://doi.org/10.3969/j.issn.1674-2346.2017.04.015

Xianru, C. (1960). Excavations of the Han Tomb of Mozuizi, Wuwei, Gansu. Archaeology, (9), 6-11.

Xiyi, Y. (1990). An overview of silk weaving and dyeing in the Tang Dynasty. Journal of Northwest University (Natural Science Edition), (3), 24-29.

Xuanping, Y. (2011). Zhe Luo. Suzhou: Soochow University Press.

Yeon Ok, S., \& Qicheng, Z. (1995). A Study on the Raw Material, Structure and Weaving Instruments of Fabrics before the West Zhou Dynasty. Journal of China Textile University (Eng. Ed.), 12(1), 49-55.

Yuchun, L. (1960). Bulletin of the Eastern Han Dynasty co-burial tombs in the burial area of the Middle Ancient Ruins in Beida Desert, Minfeng County, Xinjiang. Cultural Relics, (6), 9-12.

\section{Copyrights}

Copyright for this article is retained by the author(s), with first publication rights granted to the journal.

This is an open-access article distributed under the terms and conditions of the Creative Commons Attribution license (http://creativecommons.org/licenses/by/4.0/). 